\title{
Article
}

\section{Conjugacy Problem in the Fundamental Groups of High-Dimensional Graph Manifolds}

\author{
Raeyong Kim
}

Citation: Kim, R. Conjugacy

Problem in the Fundamental Groups of High-Dimensional Graph

Manifolds. Mathematics 2021, 9, 1330.

https://doi.org/10.3390/math9121330

Academic Editor: Adolfo

Ballester-Bolinches

Received: 26 April 2021

Accepted: 7 June 2021

Published: 9 June 2021

Publisher's Note: MDPI stays neutral with regard to jurisdictional claims in published maps and institutional affiliations.

Copyright: (C) 2021 by the author. Licensee MDPI, Basel, Switzerland. This article is an open access article distributed under the terms and conditions of the Creative Commons Attribution (CC BY) license (https:// creativecommons.org/licenses/by/ $4.0 /)$.
Department of Mathematics \& Industrial Mathematics, College of Natural and Life Sciences, Daegu University, Gyeongsan 38453, Korea; raeyongkim@daegu.ac.kr

\begin{abstract}
The conjugacy problem for a group $G$ is one of the important algorithmic problems deciding whether or not two elements in $G$ are conjugate to each other. In this paper, we analyze the graph of group structure for the fundamental group of a high-dimensional graph manifold and study the conjugacy problem. We also provide a new proof for the solvable word problem.
\end{abstract}

Keywords: high-dimensional graph manifolds; conjugacy problem; graph of groups

MSC: 20F65; 20F10; 20F67

\section{Introduction}

Since the formulation by M. Dehn on the topology of surfaces (see [1]), decision problems have played a central role in geometric group theory and combinatorial group theory. Among them, the word problem and the conjugacy problem (and the isomorphism problem) are of major importance. Focused on the fundamental groups of compact manifolds, it was M. Dehn who suggested that the fundamental groups of compact surfaces have solvable word and conjugacy problems. For dimension 3, there are various ways of proving that the fundamental groups of 3-manifolds have solvable word problems. One of the easiest ways is invoking the geometrization theorem and the work of Hempel about the residual finiteness of 3-manifold groups (see [2]). For the conjugacy problem, Préaux extended Sela's work on knot groups ([3]) to prove that the conjugacy problem is solvable in the class of the fundamental groups of 3-manifolds (see $[4,5])$. The author refers readers to [6] for decision problems for 3-manifold groups.

For the dimension $n \geq 4$, the situation is completely changed. First of all, in [7], Novikov constructed a finitely presented group with undecidable conjugacy problems. It is well known that each finitely presented group is the fundamental group of some $n$-manifold for $n \geq 4$ fixed. Therefore, the conjugacy problem is, in general, unsolvable in the class of $n$-manifolds for $n \geq 4$. Furthermore, due to the absence of the geometrization theorem, we do not expect the kind of unified arguments for the manifolds of high dimensions.

In this paper, we study algorithmic problems for the fundamental groups of recently introduced high-dimensional manifolds, which are built up with complete finite volume hyperbolic manifolds. By definition, it turns out that, as 3-manifold groups do, the fundamental groups of such manifolds have the graphs of group structure. In Section 2, we introduce the notion of high-dimensional graph manifolds. Then we study the graph of group structure for the fundamental group. It is well known that the fundamental group of the truncated complete finite volume hyperbolic manifold is a relatively hyperbolic group. In Section 3, various algorithmic problems will be discussed. As a corollary, we prove the solvability of the word problem. In the final section, we present the example of a reducible graph manifold such that the fundamental group has the unsolvable conjugacy problem and we prove the main theorem of this paper. 
Theorem 1. The fundamental group of an irreducible graph manifold has a solvable conjugacy problem.

\section{High-Dimensional Graph Manifolds}

Recently, there has been a lot of work on proving rigidity theorems for non-positively curved spaces and developing these results beyond non-positively curved spaces. In [8], Frigerio, Lafont and Sisto introduced, as a generalization of 3-manifolds supporting nonpositively curved metrics, the notion of high-dimensional graph manifolds and proved various, including topological and quasi-isometric, rigidity theorems. They also proved that there are infinitely many graph manifolds not supporting non-positively curved metrics even if graph manifolds are, by definition, built up with "pieces" supporting non-positively curved metrics, and suggested that one might need different approaches to study them to those used in studying non-positively curved spaces. For details, see [8].

In their monograph, Frigerio, Lafont and Sisto also studied various algebraic and algorithmic properties of the fundamental groups of high-dimensional graph manifolds. As indicated in the introduction, we analyze the graph of group structure on the fundamental groups of graph manifolds to study the conjugacy problem, and answer one of the questions posed in ([8], Section 12.1). The definition of a high dimensional graph manifold follows first.

Fix $n \geq 3, k \in \mathbb{N}$ and $n_{i} \in \mathbb{N}$ with $3 \leq n_{i} \leq n$ for $i=1, \cdots, k$. For every $i=$ $1, \cdots, k$, let $N_{i}$ be a complete finite-volume non-compact hyperbolic $n_{i}$-manifold with toric cusps. It is well-known that each cusp of $N_{i}$ supports a canonical smooth foliation by closed tori and defines a diffeomorphism between the cusp and $T^{n_{i}-1} \times[0, \infty)$, where $T^{n_{i}-1}=\mathbb{R}^{n_{i}-1} / \mathbb{Z}^{n_{i}-1}$ is the standard torus. Truncate the cusps of $N_{i}$ by setting $\bar{N}_{i}=$ $N_{i} \backslash \cup_{j=1}^{a_{i}} T_{j}^{n_{i}-1} \times(4, \infty)$, where $T_{j}^{n_{i}-1} \times[0, \infty), j=1, \cdots, a_{i}$ are the cusps of $N_{i}$. Let $V_{i}=$ $\bar{N}_{i} \times T^{n-n_{i}}$ and fix a subset $\mathcal{B}$ of the set of boundary components of the $V_{i}$ s. Finally, glue the $V_{i} \mathrm{~s}$ along affine diffeomorphisms between the paired tori in $\mathcal{B}$. The (connected) manifold $M$ obtained in this way is called a graph n-manifold.

The manifolds $V_{1}, \cdots, V_{k}$ are called the pieces of $M$. For every $i, \bar{N}_{i}\left(\right.$ or $\left.N_{i}\right)$ is the base of $V_{i}$ and if $p \in \bar{N}_{i}$, the set $\{p\} \times T^{n-n_{i}}$ is a fiber of $V_{i}$. The toric hypersurfaces of $M$ corresponding to the tori in $\mathcal{B}$ are called internal walls and the components of the boundary of $M$ are called boundary walls.

Definition 1. Let $M$ be a graph manifold, and $V^{+}, V^{-}$a pair of adjacent pieces of $M$. We say that two pieces have transverse fibers along the common internal wall $T$ if, under the gluing diffeomorphism $\psi: T^{+} \rightarrow T^{-}$of the paired boundary tori corresponding to $T$, the image of the fiber subgroup of $\pi_{1}\left(T^{+}\right)$under $\psi_{*}$ intersects the fiber subgroup of $\pi_{1}\left(T^{-}\right)$trivially.

Definition 2. A graph manifold is irreducible if every pair of adjacent pieces has transverse fibers along every common internal wall.

In the study of graph manifolds, irreducibility plays an important role in the sense that it enables us to understand the geometry of a graph manifold in terms of the geometry of the pieces. Using this idea, various results for quasi-isometric rigidities can be obtained (see [8], Chapter 10,11). Related to our interest, a graph manifold is called purely hyperbolic if all pieces have a trivial fiber and a purely hyperbolic graph manifold supports a nonpositively curved Riemannian metric ([8], Theorem 0.3). The fundamental group of a purely hyperbolic manifold is hyperbolic relative to cusp subgroups ([8], Theorem 0.12) and the solvable conjugacy problem ([9], III.Г. Theorem 1.4). In the case of irreducible graph manifolds, providing a much wider class than purely hyperbolic graph manifolds, relative hyperbolicity has been completely studied; namely, the fundamental group of an irreducible graph manifold is relatively hyperbolic if and only if the manifold contains a purely hyperbolic piece ([8], Proposition 6.11). The author refers readers to Osin's 
monograph ([10]) for relatively hyperbolic groups. The word problem is also solvable for the fundamental group of an irreducible graph manifold ([8], Corollary 6.32).

\section{Graph of Group Structure}

A graph of groups $(\mathcal{G}, X)$ consists of a graph $X$, a vertex group $G_{v}$ for each vertex $v$ in $X$, an edge group $G_{e}$ for each edge $e$ in $X$, and, for the edge $e$ with the initial vertex $v$, a monomorphism $i_{e}: G_{e} \rightarrow G_{v}$ from an edge group $G_{e}$ to the vertex group $G_{v}$. We also require that $G_{e}=G_{\bar{e}}$, where $\bar{e}$ is the edge $e$ with the opposite direction. For a graph of groups $(\mathcal{G}, X)$, once a base point $*$ is fixed, one defines the fundamental group $\pi_{1}(\mathcal{G}, X, *)$ of a graph of groups and it turns out that this definition is independent of the choice of a base point so that we just denote by $\pi_{1}(\mathcal{G}, X)$ (or $\pi_{1}(\mathcal{G})$, if the underlying graph is obvious). We denote $i_{e}\left(G_{e}\right)$ in $G_{v}$ by $G_{e}^{-}$and $i_{\bar{e}}\left(G_{e}\right)$ by $G_{e}^{+}$. For an element $c \in G_{e}$, denote $i_{e}(c) \in G_{e}^{-}$ by $c^{-}$and $i_{\bar{e}}(c) \in G_{e}^{+}$by $c^{+}$.

For a graph of group $(\mathcal{G}, X)$, fix the maximal tree $T$. If a vertex group has the presentation $\left\langle S_{i} \mid R_{i}\right\rangle$, then $\pi_{1}(\mathcal{G}, X)$ has the following presentation:

- Generators : $S_{1} \cup S_{2} \cup \cdots \cup S_{n} \cup\left\{t_{e} \mid e\right.$ is an edge in $\left.X\right\}$

- Relators :

$R_{1} \cup R_{2} \cup \cdots \cup R_{n} \cup\left\{\right.$ for all edge $e$, for all $\left.c \in G_{e}^{-}, t_{e}\left(i_{e}^{-1} \circ i_{\bar{e}}\right)(c) t_{e}^{-1}=c\right\}$

$\cup\left\{\right.$ for all edge $\left.e, t_{\bar{e}}=t_{e}^{-1}\right\} \cup\left\{\right.$ for all edge $\left.e \subset T, t_{e}=1\right\}$

Note that $t_{e}$ is called the stable letter associated to the edge $e$.

In this section, following [11] (Section 5), we briefly recall how to express an element in $\pi_{1}(\mathcal{G}, X)$ and prove some propositions needed for the main theorem. The author refers readers to Serre's book ([11]) for the definitions and basic properties about graphs of groups.

\subsection{Cyclically Reduced Form}

Given a graph of groups $(\mathcal{G}, X)$, a path in $X$ is a sequence $\left(v_{1}, e_{1}, v_{2}, \cdots, e_{m}, v_{m+1}\right)$, where $v_{i}$ s are vertices of $X$ and $e_{i}$ is the edge in $X$ starting from $v_{i}$ to $v_{i+1}$. The integer $m$ is the length of the path. A loop in $X$ is a path between a vertex $v$ and itself. A word of type $\mathcal{C}$ is a pair $(\mathcal{C}, \mu)$, where

- $\mathcal{C}=\left(v_{1}, e_{1}, v_{2}, \cdots, e_{m}, v_{m+1}\right)$ is a based loop in $X$.

- $\mu=\left(\mu_{1}, \cdots, \mu_{m+1}\right)$ is a sequence such that $\mu_{i}$ is an element of the vertex group $G_{v_{i}}, i=1, \cdots, m+1$.

Once a base point $*$ in $X$ is given, a word of type $\mathcal{C}$ based at $*$ defines an element in $\pi_{1}(\mathcal{G}, X, *)$, denoted by $|\mathcal{C}, \mu|, \mu_{1} t_{e_{1}} \mu_{2} t_{e_{2}} \cdots t_{e_{m}} \mu_{m+1}$. A word of type $\mathcal{C},(\mathcal{C}, \mu)$ is reduced if either its length is 0 and $v_{1} \neq 1$, or its length is $>1$ and whenever $e_{i-1}=\overline{e_{i}}$, then $\mu_{i} \notin G_{e_{i}}^{-}$.

Theorem 2 ([11], Theorem 11$)$. If $(\mathcal{C}, \mu)$ is a reduced word, then the associated element $|\mathcal{C}, \mu|$ is $\neq 1$ in $\pi_{1}(\mathcal{G}, X)$.

A cyclic conjugate of $(\mathcal{C}, \mu)=\left(\left(v_{1}, e_{1}, v_{2}, \cdots, e_{m}, v_{m+1}\right),\left(\mu_{1}, \cdots, \mu_{m+1}\right)\right)$ is a word of type $\left(\mathcal{C}^{\prime}, \mu^{\prime}\right)=\left(\left(v_{i}, e_{i}, v_{i+1}, \cdots, v_{m}, e_{m}, v_{m+1}, e_{1}, v_{2}, \cdots, e_{i-1}, v_{i}\right),\left(\mu_{i}, \cdots, \mu_{m}, \mu_{m+1} \mu_{1}, \mu_{2}\right.\right.$ $\left.\cdots, \mu_{i-1}, 1\right)$ ) for some $i, 0 \leq i \leq m$. Note that indices are taken modulo $m$. A word of type $\mathcal{C}$ is cyclically reduced if all of its cyclic conjugates are reduced, and if $\mu_{m+1}=1$ whenever $m>1$.

The fundamental group of a graph manifolds canonically has the graph of group structure induced by the decomposition of the manifold $M$ into pieces $V_{1}, \cdots, V_{k}$. More precisely, if we let $\mathcal{G}$ be the graph of groups induced by decomposition of $M$ into pieces $V_{1}, \cdots, V_{k}$, a vertex group $G_{v}$ is the fundamental group $\pi_{1}\left(V_{i}\right)$ of a piece $V_{i}$, an edge group $G_{e}$ is isomorphic to $\mathbb{Z}^{n-1}$ and a monomorphism $i_{e}: G_{e} \rightarrow G_{v}$ from an edge group $G_{e}$ into the vertex group $G_{v}$ is induced by the inclusion of a boundary component into the corresponding piece. Finally, a basic result of Bass-Serre theory implies that $\pi_{1}(M) \cong$ $\pi_{1}(\mathcal{G})$. 


\subsection{Various Algorithmic Problems}

Consider the base $\bar{N}$ of a piece of a graph manifold, which is a truncated complete finite-volume non-compact hyperbolic manifold with toric cusps. It is well known that $\pi_{1}(\bar{N})$ is hyperbolic relative to cusp subgroups. Since each peripheral subgroup is free abelian, the following algorithmic problems for the fundamental group $\pi_{1}(\bar{N})$ are all solvable. See [10] (Corollary 5.5, Theorem 5.6, Theorem 5.12).

- Word Problem. In other words, there exists an algorithm that decides, for a given $g \in \pi_{1}(\bar{N})$, whether $g$ is the identity or not.

- Conjugacy Problem.

- Membership Problem. In other words, there exists an algorithm that decides, for a given $g \in \pi_{1}(\bar{N})$ and a peripheral subgroup $T$, whether $g \in T$ or not.

- General Parabolicity Problem. In other words, there exists an algorithm that allows, for a given parabolic element $g \in \pi_{1}(\bar{N})$, finding a peripheral subgroup $T$ and $t \in \pi_{1}(\bar{N})$ such that $t^{-1} g t \in T$.

- Special Parabolicity Problem. In other words, there exists an algorithm that allows, for a given peripheral subgroup $T$ and a given element $g \in \pi_{1}(\bar{N})$ conjugate to an element in $T$, finding an element $t \in \pi_{1}(V)$ such that $t^{-1} g t \in T$.

In [8], the coarse geometry of $\pi_{1}(M)$ was used to prove that if $M$ is irreducible, $\pi_{1}(M)$ has a solvable word problem. Using the basic argument in terms of a graph of groups, we can prove that the word problem is solvable even for a reducible case.

Theorem 3. Let $M$ be a graph manifold. Then the word problem for $\pi_{1}(M)$ is solvable.

Proof. Let a word of type $\mathcal{C},(\mathcal{C}, \mu)=\left(\left(v_{1}, e_{1}, v_{2}, \cdots, e_{m}, v_{m+1}\right),\left(\mu_{1}, \cdots, \mu_{m+1}\right)\right)$ be given. Note that it defines an element $|\mathcal{C}, \mu|=\mu_{1} t_{e_{1}} \mu_{2} t_{e_{2}} \cdots t_{e_{m}} \mu_{m+1}$ in $\pi_{1}(\mathcal{G})\left(=\pi_{1}(M)\right)$. Then one can systematically reduce the word to decide whether the associated element represents the identity or not. More precisely, if there exists $i$ such that $e_{i}=\bar{e}_{i+1}$, apply the solvable membership problem to decide whether $\mu_{i+1} \in G_{e}^{+}$or not. If so, since all vertex/edge groups are finitely presented, we can find the element $\mu_{i+1}^{\prime} \in G_{e_{i}}$ such that $\mu_{i+1}^{\prime+}=\mu_{i+1}$. Then replace

$$
\left(\left(\cdots, e_{i-1}, v_{i}, e_{i}, v_{i+1}, e_{i+1}, v_{i+2}, e_{i+2} \cdots\right),\left(\cdots, \mu_{i}, \mu_{i+1}, \mu_{i+2}, \cdots\right)\right)
$$

by $\left(\left(\cdots, e_{i-2}, v_{i}, e_{i+2}, \cdots\right),\left(\cdots, \mu_{i} i_{e_{i}} \mu_{i+1}^{\prime-} \mu_{i+2}, \cdots\right)\right)$. Since this process strictly decreases the length of the loop, one must stop after finitely many such steps to obtain a word of length 0 or a reduced form. If we end up with the loop of length 0 , we apply the solvable word problem for $\pi_{1}(V)$ to determine whether it represents the identity or not. If not, by Theorem 2, the element is not the identity.

Remark 1. By using the same method as in the proof of the above theorem to every cyclic permutation, one can reduce a word of type $\mathcal{C}$ to a cyclically reduced form whose label represents some element in the same conjugacy class as the given element.

We study two algorithmic problems that are necessary to prove our main theorem. The following propositions are called the boundary parallelism problem and the two coset problem. See [4]. We denote by $g \sim h$ if $g$ and $h$ are conjugate to each other.

Proposition 1. For $k \geq 3$, let $\bar{N}$ be a truncated complete finite-volume non-compact hyperbolic $k$-manifold with toric cusps. Let $H$ be a cusp subgroup of $\pi_{1}(\bar{N})$ and $g$ be an element in $\pi_{1}(\bar{N})$. Then $C_{H}(g)=\left\{h \in H \mid g \sim h\right.$ in $\left.\pi_{1}(\bar{N})\right\}$ is empty or a singleton. Furthermore, there exists an algorithm to find $C_{H}(g)$.

Proof. It is well known that the universal cover $\widehat{\mathbb{H}}^{k}$ of $\bar{N}$ is a hyperbolic space with suitable $\pi_{1}(\bar{N})$-equivariant open horoballs removed. We consider $\pi_{1}(\bar{N})$-action on the universal 
cover. Note that the stabilizer of each horosphere corresponds to the conjugate of a cusp subgroup. Suppose that $h_{1}, h_{2} \in C_{H}(g)$. Then, by the definition of $C_{H}(g), h_{1}$ and $h_{2}$ are conjugate. Suppose that $B$ is the horosphere fixed by $H$ in $\widehat{\mathbb{H}}^{k}$ and $h_{1}=u^{-1} h_{2} u$ for some $u \in \pi_{1}(\bar{N})$. Then $u B=u h_{1}(B)=h_{2} u(B)=h_{2}(u B)$ and, since $B$ is the only horosphere fixed by $H, u B=B$. Furthermore, $\pi_{1}(\bar{N})$-action on $\widehat{\mathbb{H}}^{k}$ is free. Therefore, if $u$ fixes another horosphere so that $u$ pointwise fixes the unique minimal geodesic joining two distinct horospheres, then $u$ must be an identity. This implies that if $u$ is non-trivial, then $u$ must be parabolic and fixes $B$ so that $u \in H$. Since $H$ is free abelian, $h_{1}=h_{2}$.

Let $g \in \pi_{1}(\bar{N})$ be given. Apply the solvable membership problem to decide whether $g$ is parabolic or not. If $g$ is not parabolic, since a hyperbolic element cannot be conjugate to a parabolic element, $C_{H}(g)$ is empty. Suppose that $g$ is parabolic. Then apply the solvable general parabolicity problem to find the peripheral subgroup $T^{\prime}$ and an element $t$ such that $t^{-1} g t \in T^{\prime}$. Suppose that $T \neq T^{\prime}$. Since each conjugacy class of peripheral subgroups corresponds to an orbit of horospheres under the action of $\pi_{1}(\bar{N})$ on $\widehat{\mathbb{H}}^{k}, C_{H}(g)$ is empty. Finally, if $T=T^{\prime}$, then apply the solvable special parabolicity problem to find $C_{H}(g)$.

Note that the fundamental group $\pi_{1}(V)$ of a piece of a graph manifold is the product of $\pi_{1}(\bar{N})$ and the free abelian group. Therefore,

Corollary 1. Let $\pi_{1}(V)=\pi_{1}\left(\bar{N}^{k}\right) \times \mathbb{Z}^{n-k}$ be the fundamental group of a piece of a graph manifold. Let $\pi_{1}(T)$ be the fundamental group of a wall and $g$ be an element in $\pi_{1}(V)$. Then $C_{\pi_{1}(T)}(g)=\left\{h \in \pi_{1}(T) \mid g \sim h\right.$ in $\left.\pi_{1}(V)\right\}$ is either empty or a singleton. Furthermore, there exists an algorithm to find $C_{\pi_{1}(T)}(g)$.

Proposition 2. For $k \geq 3$, let $\bar{N}$ be a truncated complete finite-volume non-compact hyperbolic $k$-manifold with toric cusps. Let $H, H^{\prime}$ be cusp subgroups (possibly $H=H^{\prime}$ ) and $g, g^{\prime} \in \pi_{1}(\bar{N})$. Suppose further that if $H=H^{\prime}$, then $g^{\prime} \notin H$. Then $C_{H, H^{\prime}}\left(g, g^{\prime}\right)=\left\{\left(c, c^{\prime}\right) \in H \times H^{\prime} \mid g=\right.$ $\left.c g^{\prime} c^{\prime}\right\}$ is empty or a singleton. Furthermore, there is an algorithm to find $C_{H, H^{\prime}}\left(g, g^{\prime}\right)$.

Proof. Suppose that there are two distinct elements $\left(c_{1}, c_{1}^{\prime}\right),\left(c_{2}, c_{2}^{\prime}\right)$ in $C_{H, H^{\prime}}\left(g, g^{\prime}\right)$, i.e., $g=c_{1} g^{\prime} c_{1}^{\prime}, g=c_{2} g^{\prime} c_{2}^{\prime}$. Then $c_{1} g^{\prime} c_{1}^{\prime}=c_{2} g^{\prime} c_{2}^{\prime} \Rightarrow c_{2}^{-1} c_{1}=g^{\prime} c_{2}^{\prime}\left(c_{1}^{\prime}\right)^{-1}\left(g^{\prime}\right)^{-1}$. This implies that $c_{2}^{-1} c_{1} \in H$ is conjugate to $c_{2}^{\prime}\left(c_{1}^{\prime}\right)^{-1} \in H^{\prime}$. Note that elements in two distinct cusp subgroups cannot be conjugate. Applying the same idea as Proposition 1 concludes that $c_{2}^{-1} c_{1}=c_{2}^{\prime}\left(c_{1}^{\prime}\right)^{-1}=1$. Therefore, $C_{H, H^{\prime}}\left(g, g^{\prime}\right)$ is at most a singleton.

By [4] (Lemma 6.1), there exists a constant $K$ that depends on the length of $g$ and $g^{\prime}$ and constants related to the relative hyperbolicity of $\pi_{1}(\bar{N})$ such that if $g=c_{1} g^{\prime} c_{2}$, then $c_{1}$ and $c_{2}$ have the length at most $K$. Apply the solvable word problem to find all pairs $\left(c_{1}, c_{2}\right)$ such that $g=c_{1} g^{\prime} c_{2}$. Then use the solvable membership problem to determine whether $c_{1} \in H$ and $c_{2} \in H^{\prime}$.

Corollary 2. Let $\pi_{1}(V)=\pi_{1}\left(\bar{N}^{k}\right) \times \mathbb{Z}^{n-k}$ be the fundamental group of a piece of graph manifold. Let $\pi_{1}(T), \pi_{1}\left(T^{\prime}\right)$ be the fundamental groups of walls and $g, g^{\prime}$ be an element in $\pi_{1}(V)$. Then $C_{\pi_{1}(T), \pi_{1}\left(T^{\prime}\right)}\left(g, g^{\prime}\right)=\left\{\left(h, h^{\prime}\right) \in \pi_{1}(T) \times \pi_{1}\left(T^{\prime}\right) \mid g=h g^{\prime} h^{\prime}\right\}$ is either empty or a singleton. Furthermore, there exists an algorithm to find $C_{\pi_{1}(T), \pi_{1}\left(T^{\prime}\right)}\left(g, g^{\prime}\right)$.

\section{Conjugacy Problem in Fundamental Groups of Graph Manifolds}

In this section, we present the algorithm proving the main theorem. Note that for the graph manifold, being irreducible is necessary for the solvable conjugacy problem. Before we proceed, we give an example of reducible graph manifolds such that the conjugacy problem is unsolvable.

Consider the following graph of groups $(\mathcal{G}, X): X$ consists of 2 vertices $v, v^{\prime}$ and $(p+1)$-edges $e_{0}, e_{1}, \cdots, e_{p}$ for some $p \geq 1$. All the vertex groups and edge groups are $\mathbb{Z}^{4}$ Finally, for the edge $e_{0}$, the monomorphism from $G_{e_{0}}$ to $G_{i\left(e_{0}\right)}$ is the identity, where $i\left(e_{0}\right)$ is 
the initial vertex of the edge $e_{0}$. For $i=1,2, \cdots, p$, and each edge $e_{i}$, the monomorphism from the edge group $G_{e_{i}}$ to the initial vertex group $G_{i\left(e_{i}\right)}$ is some affine map $\phi_{i} \in \operatorname{GL}(4, \mathbb{Z})$.

Proposition 3. For the graph of groups $(\mathcal{G}, X)$ above, two elements $g$ and $g^{\prime}$ in vertex groups are conjugate if and only if there exists $\phi \in\left\langle\phi_{1}, \phi_{2}, \cdots, \phi_{p}\right\rangle \leq \mathrm{GL}(4, \mathbb{Z})$ such that $g=\phi\left(g^{\prime}\right)$.

Proof. Choose the subgraph $T$ consisting of two vertices and the edge $e_{0}$ as the maximal tree. Suppose that $g$ is conjugate to $g^{\prime}$ by $k \in \pi_{1}(\mathcal{G})$, i.e., $g=k^{-1} g^{\prime} k$. Let $(\mathcal{C}, \mu)=$ $\left(\left(v_{1}, e_{1}, v_{2}, \cdots, e_{m}, v_{m+1}\right),\left(\mu_{1}, \cdots, \mu_{m+1}\right)\right)$ be the type of word $\mathcal{C}$ whose label represents $k$. Then

$$
k^{-1} g^{\prime} k=\left(\mu_{m+1}\right)^{-1}\left(t_{e_{m}}\right)^{-1} \cdots\left(t_{e_{1}}\right)^{-1}\left(\mu_{1}\right)^{-1} g^{\prime} \mu_{1} t_{e_{1}} \cdots t_{e_{m}} \mu_{m+1}
$$

Consider $\left(\mu_{1}\right)^{-1} g^{\prime} \mu_{1}$ in the middle. If $\mu_{1}$ and $g^{\prime}$ are contained in the distinct vertex groups, use the edge $e_{0}$ to replace $g^{\prime}$ by the same element that is contained in the same vertex group as $\mu_{1}$. Then, since the vertex group is free abelian, $\left(\mu_{1}\right)^{-1} g^{\prime} \mu_{1}=g^{\prime}$. Use the same idea and the relation $\left(t_{e_{i}}\right)^{-1} h t_{e_{i}}=\phi_{e_{i}}(h)$. Then we can prove that there exists $\phi \in\left\langle\phi_{1}, \phi_{2}, \cdots, \phi_{p}\right\rangle$ such that $g=\phi\left(g^{\prime}\right)$. The opposite direction is obvious.

In [12], Bogopolski, Martino and Ventura proved that there exists a finitely generated subgroup $H=\left\langle\phi_{1}, \cdots, \phi_{p}\right\rangle$ of $\mathrm{GL}(4, \mathbb{Z})$ such that it is impossible to decide whether two elements of $\mathbb{Z}^{4}$ are in the same orbit under the action of $H$. Using this fact, we can provide the example of a reducible graph manifold $M$ such that $\pi_{1}(M)$ has an unsolvable conjugacy problem.

Let $\bar{N}$ be a truncated complete finite-volume non-compact hyperbolic manifold with toric cusps. Suppose that $\bar{N}$ has $(p+1)$ cusps. Let $V$ be the product of $\bar{N}$ and a 4 dimensional torus and $D V$ be the graph manifold obtained by gluing the cusps of two copies of $V$ as follows. One pair of cusps is glued by the map that induces the identity on the fundamental groups. The other $p$-pairs of cusps are glued by the maps each of which induces the affine map, for $i=1,2, \cdots, p$,

$$
\left(\begin{array}{cc}
\text { Id. } & 0 \\
0 & \phi_{i}
\end{array}\right)
$$

Note that the identity part above means that we glue the cusp of $\bar{N}$ to the cusp of the other piece by the map inducing the identity on the fundamental groups and $\phi_{i} \in \mathrm{GL}(4, \mathbb{Z})$ corresponds to the gluing of two 4-dimensional tori, one for each copy.

Theorem 4. The fundamental group $\pi_{1}(D V)$ of the graph manifold described above has an unsolvable conjugacy problem.

Proof. By [12] and Proposition 3, it is impossible to determine whether two elements in the fibers are conjugate or not.

Remark 2. Contrary to the low-dimensional cases, it is not easy to construct hyperbolic manifolds in high dimensions. This is basically due to the absence of the geometrization theorem. Nevertheless, in dimensional 3, Thurston constructed various hyperbolic manifolds with arbitrary numbers of cusps and this eliminates the existence issue of the example above. See [13] for details.

We present the algorithm to prove the main theorem. The algorithm uses the following theorem, which is well known to experts. The proof can be found in [4].

Theorem 5 ([4], Theorem 3.1). Suppose $(\mathcal{C}, \mu)$ and $\left(\mathcal{C}^{\prime}, \mu^{\prime}\right)$ are cyclically reduced forms, whose labels $w$ and $w^{\prime}$ are conjugate in the fundamental group of a graph of groups $\pi_{1}(\mathcal{G}, X)$. Then $(\mathcal{C}, \mu)$ and $\left(\mathcal{C}^{\prime}, \mu^{\prime}\right)$ have the same length, and moreover, either

1. their length is equal to $0, \mathcal{C}=\mathcal{C}^{\prime}=\left(v_{\sigma_{1}}\right)$, and $w$ and $w^{\prime}$ are conjugate in $G_{v_{\sigma_{1}}}$; 
2. their length is equal to 0 , and there exists a path $\left(v_{\alpha_{0}}, e_{\beta_{1}}, \cdots, e_{\beta_{p}}, v_{\alpha_{p}}\right)$ in $X$, and a sequence $\left(c_{1}, c_{2}, \cdots, c_{p}\right)$ with $\forall i=1,2, \cdots, p, c_{i}$ lying in the edge group $G_{e_{\beta_{i}}}$, such that $w \in$ $G_{v_{\alpha_{0}}}, w^{\prime} \in G_{v_{\alpha_{p}}}$, and

$$
\begin{aligned}
& w \sim c_{1}^{-} \text {in } G_{v_{\alpha_{0}}} \\
& w^{\prime} \sim c_{p}^{+} \text {in } G_{v_{\alpha_{p}}} \\
& \text { and } \forall i=1,2, \cdots, p-1, c_{i}^{+} \sim c_{i+1}^{-} \text {in } G_{v_{\alpha_{i}}} \text {;or }
\end{aligned}
$$

3. their length is greater than 0. Up to the cyclic permutation of $\left(\mathcal{C}^{\prime}, \mu^{\prime}\right)$, the loops $\mathcal{C}, \mathcal{C}^{\prime}$ are equal, $\mathcal{C}=\mathcal{C}^{\prime}=\left(v_{\sigma_{1}}, e_{\tau_{1}}, \cdots, v_{\sigma_{n}}, e_{\tau_{n}}\right)$, and there exists a sequence $\left(c_{1}, \cdots, c_{n}\right)$, with all $i=1, \cdots, n, c_{i}$ lying in the edge group $G_{e_{\tau_{i}}}$ such that

$$
\begin{aligned}
& \mu_{1}=c_{n}^{+} \mu_{1}^{\prime}\left(c_{1}^{-}\right)^{-1} \text { in } G_{v_{\sigma_{1}}} \\
& \forall i=2,3, \cdots, n, \mu_{i}=c_{i-1}^{+} \mu_{i}^{\prime}\left(c_{i}^{-}\right)^{-1} \text { in } G_{v_{\sigma_{i}}}
\end{aligned}
$$

In particular, the element $c_{n}^{+} \in G_{e_{\tau_{n}}}^{+}$conjugates $w^{\prime}$ into $w$ in $\pi_{1}(\mathcal{G}, X)$ :

$$
w=c_{n}^{+} w^{\prime}\left(c_{n}^{+}\right)^{-1} \text { in } \pi_{1}(\mathcal{G}, X)
$$

Definition 3. Let $G$ be the fundamental group of a graph of groups $\mathcal{G}$. We say G-action on the Bass-Serre tree $T$ associated to $\mathcal{G}$ is $K$-acylindrical if there exists a constant $K$ such that any element that pointwise fixes any path in $T$ of length $\geq K$ is automatically trivial. The action is acylindrical if it is K-acylindrical for some $K$.

Proposition 4 ([8], Proposition 6.4). If $M$ is irreducible, then $\pi_{1}(M)$-action on the Bass-Serre tree $T$ is 3-acylindrical.

Proposition 5. Let $M$ be an irreducible graph manifold and $u, v \in \pi_{1}(M)$ be conjugate elements lying in vertex groups. Then any reduced sequence in Theorem 5 has length at most 2.

Proof. Let $\widetilde{e}_{1}, \widetilde{e}_{2}$ be two consecutive edges sharing the vertex $\widetilde{v}_{1}$, and $\widetilde{c}_{1} \in G_{\widetilde{e}_{1}}, \widetilde{c}_{2} \in G_{\widetilde{e}_{2}}$ be elements in edge groups in the Bass-Serre tree. If $\widetilde{c}_{1}^{+}$is conjugate to $\widetilde{c}_{2}^{-}$by $k \in G_{\widetilde{v}_{1}}$, i.e., $\widetilde{c}_{1}^{+}=k^{-1} \widetilde{c}_{2}^{-} k$, then for the wall $B$ corresponding to the starting vertex of the edge $\widetilde{e}_{2}$, $\widetilde{c}_{1}^{+}\left(k^{-1} B\right)=k^{-1} \widetilde{c}_{2}^{-} k\left(k^{-1} B\right)=k^{-1} B$. This implies that $\widetilde{c}_{1}^{+}$fixes the edge $\widetilde{e}_{1}$ and $k \widetilde{e}_{2}$.

Suppose $\left(v_{\alpha_{0}}, e_{\beta_{1}}, \cdots, e_{\beta_{p}}, v_{\alpha_{p}}\right)$ is the reduced path and $\left(c_{1}, c_{2}, \cdots, c_{p}\right)$ be the reduced sequence that appeared in Theorem 5. If we consider the lift of the path in the Bass-Serre tree, then, by the argument above and [8] (Lemma 6.3), each $c_{i}$ must be contained in the fiber subgroup of $G_{v_{\alpha_{i}}}$. Since $M$ is irreducible, $p$ must be less than 3 .

Theorem 6. The fundamental group of an irreducible graph manifold $M$ has a solvable conjugacy problem.

Proof. Suppose that we are given two words $w$ and $w^{\prime}$. Find words of type $\mathcal{C}$ whose labels are $w$ and $w^{\prime}$ and reduce them to cyclically reduced forms. Note that a cyclically reduced form represents an element in the conjugacy class. Without loss of generality, let $(\mathcal{C}, \mu)$ and $\left(\mathcal{C}^{\prime}, \mu^{\prime}\right)$ be cyclically reduced words that label precisely $w$ and $w^{\prime}$. By Theorem 5 , we assume that they have the same length, otherwise $w$ and $w^{\prime}$ are not conjugate. We have the following two cases.

- $\quad$ Suppose that $(\mathcal{C}, \mu)$ and $\left(\mathcal{C}^{\prime}, \mu^{\prime}\right)$ have the length of 0 . Let us say that $\mathcal{C}=(v)$ and $\mathcal{C}=\left(v^{\prime}\right)$. If $v=v^{\prime}$, apply the solvable conjugacy problem to $G_{v}$ to determine whether $w$ is conjugate to $w^{\prime}$ in $G_{v}$. Note that $G_{v}$ is isomorphic to the product of $\pi_{1}(\bar{N})$ and the free abelian group. Since $\pi_{1}(\bar{N})$, as a relatively hyperbolic group, has a solvable conjugacy problem, so does $G_{v}$. If $w$ and $w^{\prime}$ are conjugate in $G_{v}$, then conclude that they are conjugate in $\pi_{1}(M)$. If not, we do not conclude anything yet and proceed to the following general case. For each boundary subgroup $T$ of $G_{v}$, use the solution in 
Corollary 1 to find an element $c^{-}$in $T$ conjugate to $w$ in $G_{v}$. Note that such elements are at most singleton. Repeat the same process for each boundary subgroup of $G_{v^{\prime}}$. Now for the vertex group containing $c^{+}$and each boundary subgroup, find an element in the boundary subgroup conjugate to $c^{+}$in the vertex group. Repeat the process to successively obtain the conjugate elements in the boundaries. At each step, by Corollary 1, there exists at most one conjugate element. Furthermore, since $M$ is assumed to be irreducible, by Proposition 5, this process must stop at finitely many steps. By Theorem 5, we can determine whether $w$ is conjugate to $w^{\prime}$ or not.

- Suppose that $(\mathcal{C}, \mu)$ and $\left(\mathcal{C}^{\prime}, \mu^{\prime}\right)$ both have the length $>0$. Up to cyclic permutations, we can assume that $\mathcal{C}$ and $\mathcal{C}^{\prime}$ are the same loop, otherwise $w$ is not conjugate to $w^{\prime}$, by Theorem 5 . Suppose that $\mathcal{C}=\mathcal{C}^{\prime}=\left(v_{1}, e_{1}, \cdots, v_{n}, e_{n}\right)$. For the boundary subgroups in $G_{v_{1}}$ corresponding to the edge $e_{n}$ and $e_{1}$, use the solution in Corollary 2 to find element $c_{n} \in G_{e_{n}}, c_{1} \in G_{e_{1}}$ such that $\mu_{1}=c_{n}^{+} \mu_{1}^{\prime}\left(c_{1}\right)^{-1}$ in $G_{v_{1}}$. Note that there exists at most one such pair of elements. If such $c_{n}^{+}$exists, apply the solvable word problem of $\pi_{1}(M)$ (Theorem 3) to determine $w=c_{n}^{+} w^{\prime}\left(c_{n}^{+}\right)^{-1}$ or not. If so, conclude that $w$ is conjugate to $w^{\prime}$ in $\pi_{1}(M)$. If not, apply the same process to all possible cyclic conjugates $\left(\mathcal{C}^{\prime \prime}, \mu^{\prime \prime}\right)$ of $\left(\mathcal{C}^{\prime}, \mu^{\prime}\right)$ such that $\mathcal{C}^{\prime}=\mathcal{C}^{\prime \prime}$. Since there are finitely many such conjugates, the process must stop and we can finally determine whether $w$ and $w^{\prime}$ are conjugate in $\pi_{1}(M)$.

Funding: This work was supported by the National Research Foundation of Korea (NRF) grant funded by the Korean government (MSIP; Ministry of Science, ICT \& Future Planning) (No. NRF2017R1C1B5076543).

Conflicts of Interest: The author declares no conflict of interest.

\section{References}

1. Dehn, M. Über unendliche diskontinuierliche Gruppen. Mathematische Annalen 1911, 71, 116-144. [CrossRef]

2. Hempel, J. Residual finiteness for 3-manifolds. In Combinatorial Group Theory and Topology.(AM-111), Volume 111; Princeton University Press: Princeton, NJ, USA, 2016; pp. 379-396.

3. Sela, Z. The conjugacy problem for knot groups. Topology 1993, 32, 363-369. [CrossRef]

4. Préaux, J.P. Conjugacy problem in groups of oriented geometrizable 3-manifolds. Topology 2006, 45, 171-208. [CrossRef]

5. Préaux, J.P. The conjugacy problem in groups of non-orientable 3-manifolds. Groups Geometry Dynam. 2016, 10, 473-523. [CrossRef]

6. Aschenbrenner, M.; Friedl, S.; Wilton, H. Decision problems for 3-manifolds and their fundamental groups. Geometry Topol. Monogr. 2015, 19, 201-236.

7. Novikov, P.S. Unsolvability of the conjugacy problem in the theory of groups. Izvestiya Rossiiskoi Akademii Nauk. Seriya Matematicheskaya 1954, 18, 485-524.

8. Frigerio, R.; Lafont, J.F.; Sisto, A. Rigidity of High Dimensional Graph Manifolds; Asterisque Series; Société mathématique de France: Paris, France, 2015.

9. Bridson, M.R.; Haefliger, A. Metric Spaces of Non-Positive Curvature; Springer Science \& Business Media: Berlin/Heidelberg, Germany, 2013; Volume 319.

10. Osin, D. Relatively Hyperbolic Groups: Intrinsic Geometry, Algebraic Properties, and Algorithmic Problems; American Mathematical Society, American Mathematical Society: Providence, RI, USA, 2006.

11. Stilwell, J.; Serre, J.P. Trees; Springer Monographs in Mathematics, Springer: Berlin/Heidelberg, Germany, 2013.

12. Bogopolski, O.; Martino, A.; Ventura, E. Orbit decidability and the conjugacy problem for some extensions of groups. Trans. Am. Math. Soc. 2010, 362, 2003-2036. [CrossRef]

13. Thurston, W.P. The Geometry and Topology of Three-Manifolds; Princeton University Princeton: Princeton, NJ, USA, 1979. 